\title{
The Comparison Between the Graded Photonic Crystal Coupler and Various Couplers
}

\author{
Hung-Ta Chien, Chengkuo Lee, Member, IEEE, Hua-Kung Chiu, Kuei-Chu Hsu, Chii-Chang Chen,
} Ja-an Annie Ho, and Chien Chou

\begin{abstract}
The coupling between a dielectric waveguide and a photonic crystal waveguide is studied theoretically and experimentally. The coupling methods studied in this work include graded photonic crystal coupler, photonic crystal taper, waveguide taper, and parabolic mirror coupler. The properties of each coupler are discussed and compared.
\end{abstract}

Index Terms-Integrated optics, optical couplers.

\section{INTRODUCTION}

$\mathbf{P}$ HOTONIC crystals (PCs) have outstanding ability to control the behavior of electromagnetic (EM) waves, because EM waves cannot propagate in a PC if its frequency is in the forbidden band gap. Introducing defects in PCs can realize many compact optical devices [1]-[5]. Most of these devices are embedded in PCs, so photonic crystal waveguides (PCWGs) may be necessary to guide and route the EM waves in PCs from the outside to the devices. A PCWG is a line defect in a PC [1], [2], and EM waves can be localized around the line defect if its frequency is in the forbidden band gap and the dimension of the defect is proper. The widths of a PCWG and a dielectric waveguide (WG) are usually around one and several wavelengths, respectively. This significant difference makes the low coupling efficiency between a PCWG and a dielectric WG.

Several methods have been studied to improve the coupling efficiency [6]-[11]. In this paper, a graded PC is used to alter the mode of a dielectric WG to that of a PCWG leading to reduce the coupling loss. The graded PC is a deformed PC that consists

\footnotetext{
Manuscript received September 18, 2008; revised December 01, 2008. First published April 24, 2009; current version published July 01, 2009. This work was supported in part by the National University of Singapore, Singapore, under Research Grants URC R-263-000-475-112 and FRC R-263-000-358-112/133.

H.-T. Chien, H.-K. Chiu, and C.-C. Chen are with the Department of Optics and Photonics, National Central University, Jhongli 32001, Taiwan (e-mail: htchien@ ios.ncu.edu.tw; s9424004@ios.ncu.edu.tw; trich@ dop.ncu.edu.tw).

C. Lee is with the Department of Electrical and Computer Engineering, National University of Singapore, Singapore 117576, and also with the Institute of Microelectronics (IME), Agency for Science, Technology, and Research (A* STAR), Singapore 117685 (e-mail: elelc@nus.edu.sg).

K.-C. Hsu is with the Graduate Institute of Electro-Optical Engineering, Chang Gung University, 259 Tao-Yuan, Taiwan (e-mail: jessica. eo91g@nctu.edu.tw).

J.-a. A. Ho is with the BioAnalytical Laboratory, Department of Chemistry, National Tsing Hua University, Hsinchu, 300 Taiwan (e-mail: jaho@mx.nthu. edu.tw).

C. Chou is with the Department of Biomedical Imaging and Radiological Science, and Institute of Biophotonics, National Yang Ming University, Taipei 112, Taiwan (e-mail: cchou@ym.edu.tw).

Color versions of one or more of the figures in this paper are available online at http://ieeexplore.ieee.org.

Digital Object Identifier 10.1109/JLT.2008.2012271
}

of spatial-varying air hole array. In our previous work [12], a graded PC had been designed to focus a collimated EM wave, and the spot size can be around only two lattice constants. The spot size is very close to the modal width of a single-line-defect PCWG, which show that the graded PC is an excellent candidate for the coupler between a dielectric WG and a PCWG. In this paper, we study theoretically and experimentally the coupling efficiency for several couplers including the PC taper [6]-[8], the WG taper [9], [10] and the parabolic mirror coupler (J-coupler) [11]. The design of the couplers in this work is very simple. The radii of the air holes in the graded PC coupler are linearly reduced from the edge column to the central column. In the PC taper, the width of taper is reduced step by step. Equal step size between the individual columns of the PC taper is introduced. In the WG taper, the width of taper is linearly reduced. In the parabolic mirror coupler, the curvature of mirror is a fraction of the parabola. Although there are many improving methods for the couplers, we only studied the innate properties of the couplers here.

\section{Device Structure AND Simulation}

\section{A. Device Structure and Simulation Method}

All of the devices were fabricated on a silicon-on-insulator substrate. The thickness of the silicon device layer and the insulator layer are $400 \mathrm{~nm}$ and $2 \mu \mathrm{m}$, respectively. In order to increase the coupling efficiency between the input single-mode fiber to the dielectric WGs, the width of the dielectric WG ( $W_{\mathrm{WG}}$ ) was chosen as $W_{\mathrm{WG}}=10 \mu \mathrm{m}$. The PCWG is a single-line defect along $\Gamma-\mathrm{K}$ direction in the PC formed by air holes arranged in hexagonal lattice. The lattice constant of the PC is $620 \mathrm{~nm}$ and the diameter $(D)$ of the air holes is $D=533 \mathrm{~nm}$. The corresponding forbidden band gap is between $\lambda=1320 \mathrm{~nm}$ to $2140 \mathrm{~nm}$ for the TE mode (electric field parallel to the sample surface). Thus, we choose the wavelength of the EM waves at $\lambda=1550 \mathrm{~nm}$ and the TE polarization which is around the center of the forbidden band gap of the PC.

The finite-difference time-domain (FDTD) method [13] is adopted to study the coupling efficiency theoretically. Because three-dimensional calculations need enormous memory and computation time, the structure is reduced to two-dimensional by the effective index method. The effective index of the fundamental TE mode of the silicon layer with a thickness of $400 \mathrm{~nm}$ is $n_{\text {eff }}=3.17$.

The graded PC coupler consists of $25 \times 10$ air holes in rectangular lattice as designed in our previous work. [12] The air-hole diameters of the central column and the edge column of the 


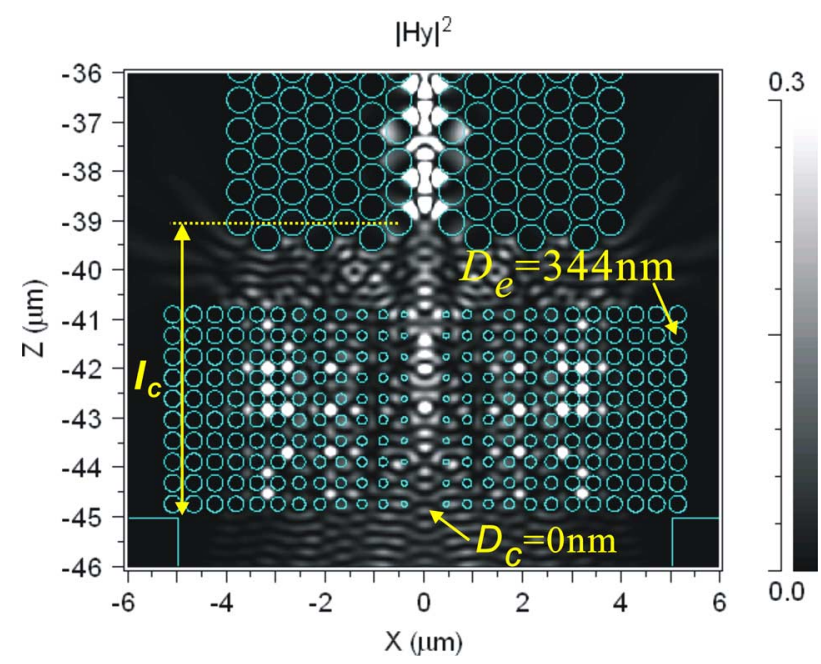

Fig. 1. Structures and energy density distributions of the graded PC coupler $\left(l_{c}=6 \mu \mathrm{m}\right)$.

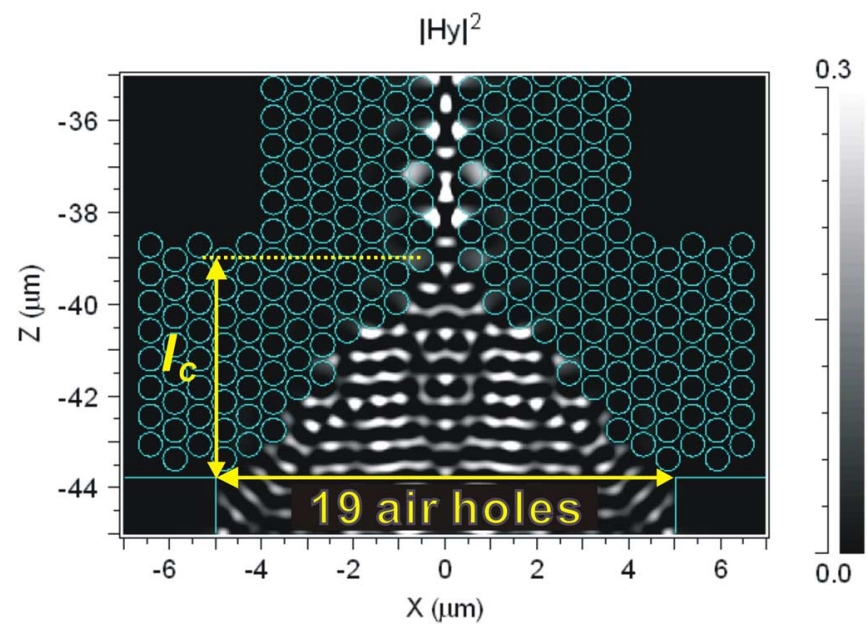

Fig. 2. Structures and energy density distributions of the PC taper $\left(l_{c}=5 \mu \mathrm{m}\right)$.

graded PC are chosen to be $D_{c}=0 \mu \mathrm{m}$ and $D_{e}=344 \mu \mathrm{m}$, respectively. The diameters are increased from the central to the edge column as shown in Fig. 1. We vary the coupling length $\left(l_{c}\right)$ which is defined as the distance between the output end of the dielectric WG and the input end of the PCWG from $l_{c}=5 \mu \mathrm{m}$ to $14 \mu \mathrm{m}$.

The simple PC taper is a horn structure formed by air hole array. The diameter of the air holes is $D=533 \mathrm{~nm}$. The width of the PC taper is reduced from $10 \mu \mathrm{m}$ (19 air holes) to $738 \mathrm{~nm}$ (one air hole) in a taper length of $l_{c}=5 \mu \mathrm{m}$ is shown in Fig. 2. The taper length is varied from $l_{c}=5 \mu \mathrm{m}$ to $30 \mu \mathrm{m}$ to study the coupling efficiency

The WG taper is a dielectric WG whose width is linearly reduced to match the optical mode of the PCWG as shown in Fig. 3. The taper length is varied from $l_{c}=5 \mu \mathrm{m}$ to $50 \mu \mathrm{m}$.

A parabolic mirror can focus the EM waves propagating parallel to the axis of the parabola to the focus of the parabola. The structure to couple the EM waves from the dielectric WG to a PCWG is shown in Fig. 4. The principle of the design is to have the energy flow of the EM waves at the focal point propagate in

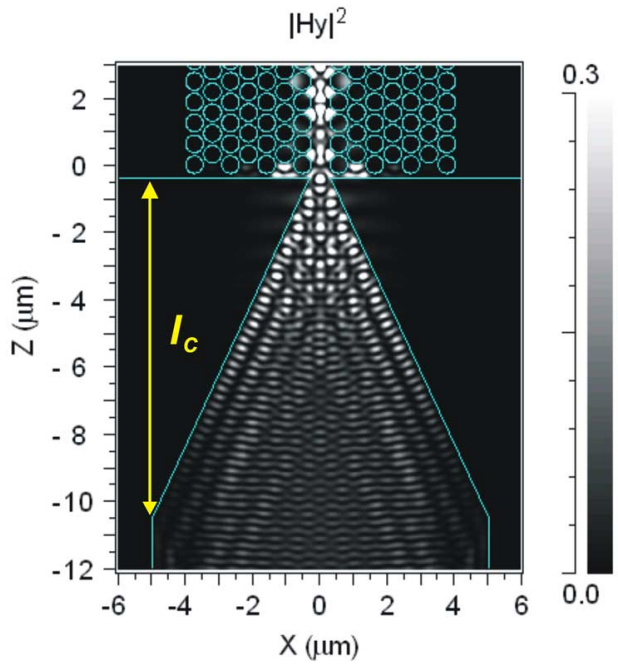

Fig. 3. Structures and energy density distributions of the WG taper $\left(l_{c}=\right.$ $10 \mu \mathrm{m})$.

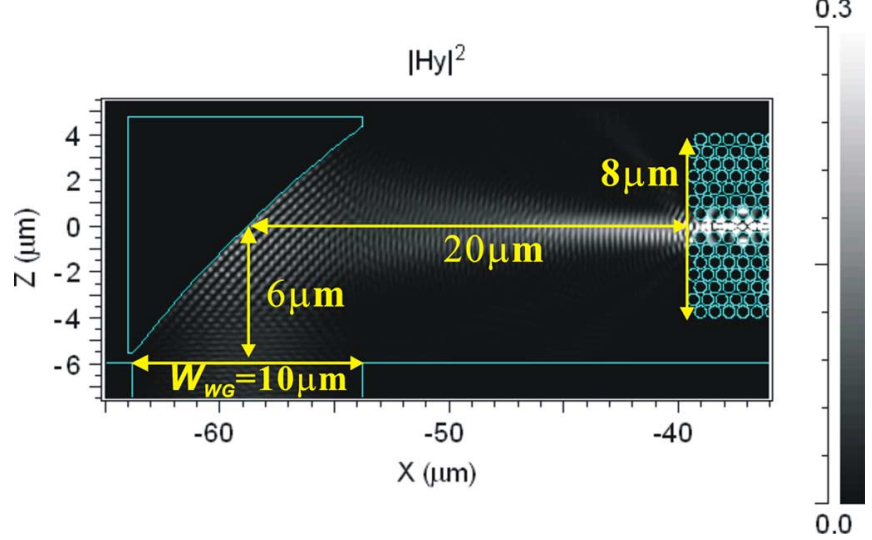

Fig. 4. Structures and energy density distributions of the parabolic mirror coupler $\left(l_{c}=26 \mu \mathrm{m}\right)$.

only the horizontal direction. [11] The EM waves are reflected by the parabolic mirror with a $90^{\circ}$ angle. The input end of the PCWG is positioned at the focus of the parabola. The coupling length can be regarded as the propagating distance of the EM waves between the output end of the dielectric WG and the input end of the PCWG. In Fig. 4, the coupling length is $l_{c}=26 \mu \mathrm{m}$. Due to the geometric constrain for design, the coupling length of the parabolic mirror coupler should be longer than the width of the dielectric WG $\left(W_{\mathrm{WG}}=10 \mu \mathrm{m}\right)$ or that of the PC structure $(8 \mu \mathrm{m})$. In this case, the parabolic mirror coupler with a coupling length shorter than around $8 \mu \mathrm{m}$ is not possible to be realized. This constrain limits us to compare the coupling efficiency of the parabolic mirror coupler with the results obtained from the graded PC which can provide a coupling length shorter than $8 \mu \mathrm{m}$. In general, an optical system design with low numerical aperture (NA) (longer coupling length) could provide longer depth of focus and require lower fabrication resolution leading to lower discrepancy between the design and the fabrication. Therefore, in this study, we design only one parabola mirror coupler with a long coupling length to be $l_{c}=26 \mu \mathrm{m}$ 

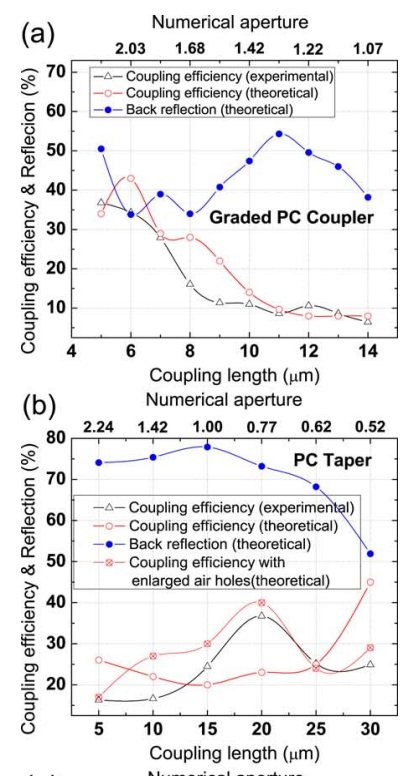

(c)
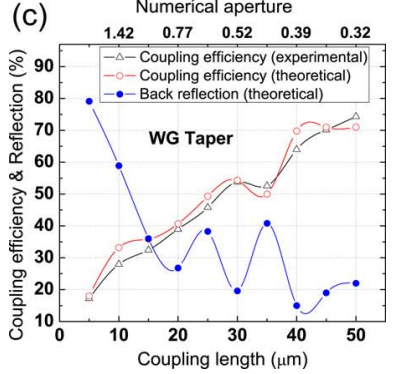

Fig. 5. Coupling efficiencies and back reflections of (a) graded PC coupler, (b) PC taper, and (c) WG taper.

( $N A=0.60)$ to provide one coupling efficiency result to compare with the other couplers. The NA is defined as

$$
N A=n_{\mathrm{eff}} \frac{W_{\mathrm{WG}}}{2 \sqrt{l_{c}^{2}+\left(\frac{W_{\mathrm{WG}}}{2}\right)^{2}}} .
$$

\section{B. Simulation Results}

The original coupling efficiency is only $5.6 \%$ if the flat input WG ending directly connects to the PCWG. For the couplers, the relations between the coupling efficiencies, back reflections, coupling length and numerical aperture from FDTD calculations are shown in Fig. 5. The coupling efficiency of the graded PC coupler is shown in Fig. 5(a). We can find that the coupling efficiency is decreased as increasing the coupling length. The maximum coupling efficiency is around $43 \%$ as the coupling length is $l_{c}=6 \mu \mathrm{m}(N A=2.03)$.

Fig. 5(b) shows the result of PC taper. The maximum coupling efficiency of $45 \%$ is obtained as the taper length is $l_{c}=$ $30 \mu \mathrm{m}(N A=0.52)$, and the back reflection is quite high here.

Fig. 5(c) shows the result of WG taper, we find that the coupling efficiency is increased with increasing the taper length. The maximum coupling efficiency is $71 \%$ for the taper length of $l_{c}=50 \mu \mathrm{m}(N A=0.32)$.

For the parabolic mirror coupler, the coupling efficiency is $78 \%$ in theoretical result.

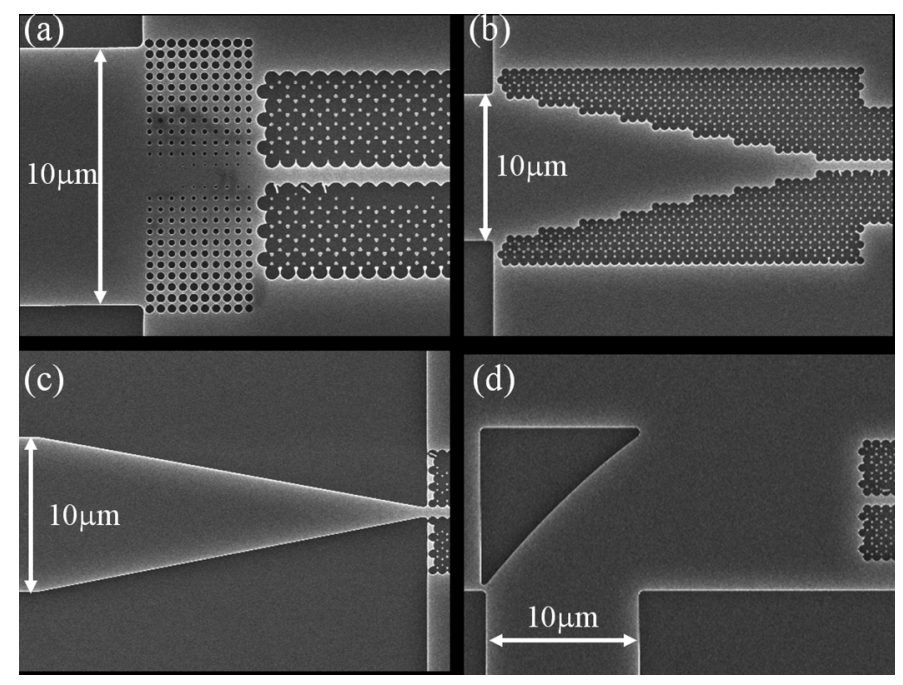

Fig. 6. SEM images of (a) the graded PC coupler, (b) PC taper, (c) WG taper, and (d) parabolic mirror coupler.

\section{FABRICATIONS, MEASUREMENT, AND DisCUSSION}

\section{A. Fabrications and Measurement}

The devices were fabricated by deep UV photolithography and silicon reactive ion etching techniques. Fig. 6 shows the scanning electron microscope (SEM) images of the devices. We can find that the air holes of the PCWG are merged together because of over etching in fabrication process. The diameter of the air holes is enlarged from $D=533 \mathrm{~nm}$ to $632 \mathrm{~nm}$. The complete band gap does not exist in the structure reducing the horizontal confinement of EM waves in the PCWG and increasing the scattering loss. Plane wave expansion calculations have been done to ensure the fact that this deformed PCWG can still guide the EM waves for the wavelength at $\lambda=1550 \mathrm{~nm}$. The measured propagation loss of the PCWG is $0.095 \mathrm{~dB} / \mu \mathrm{m}$. The diameter of the air hole in the PC taper is also enlarged leading to a significant difference between the experimental and theoretical results in the case of PC taper as shown in Fig. 5(b).

The 1-dB passband width was also studied for the couplers with the highest coupling efficiency. The passband width of the graded PC coupler and the WG taper are 7.3 and $8.6 \mathrm{~nm}$, respectively. For the other couplers, the results show narrower passband width $(<5 \mathrm{~nm})$. The passband width could be increased through optimization.

In measurement, butt-coupling method was adopted to couple the light between a single mode fiber and the dielectric WGs. The polarization was controlled to be TE polarization. In our design, PCWGs with different length was fabricated to calculate the propagation loss. (The lengths of the PCWG are around $156 \mu \mathrm{m}$ and $40 \mu \mathrm{m}$.) The coupling loss of the couplers can be obtained by subtracting all the loss except the coupling loss of the coupler from total loss of the devices.

\section{B. Measurement Results and Discussion}

The experimental results are also shown in Fig. 5. Fig. 5(a) shows that the maximum coupling efficiency of the graded PC is $37 \%$ for $l_{c}=5 \mu \mathrm{m}(N A=2.24)$. For this coupling length, this coupling efficiency is the highest for all couplers studied 


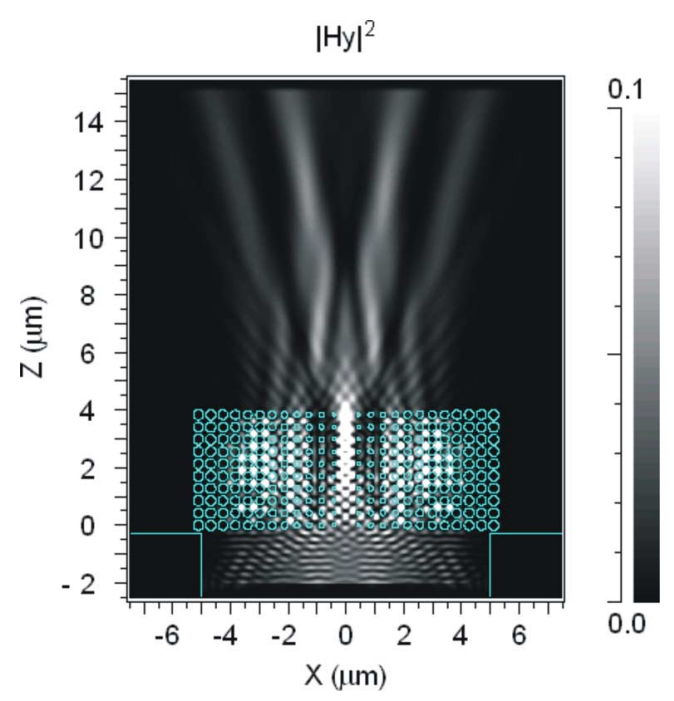

Fig. 7. Energy density distributions of the graded PC coupler.

in this work. Fig. 7 shows the energy density distribution when the EM waves passing through the graded PC coupler without attaching the PCWG. We can find that the focal length is around $5 \sim 6 \mu \mathrm{m}$ which agrees with the experimental result.

The experimental results of PC taper are shown in Fig. 5(b). The highest coupling efficiency is $37 \%$ when the coupling length is $l_{c}=20 \mu \mathrm{m}(N A=0.77)$. The lattice constant and the diameter of the air holes of the photonic crystal taper are identical to those of the PCWG. The diameter of the air holes is also enlarged after the fabrication leading to the lack of the PC confinement. Additionally, the edge of the PC taper is not smoother than that of the WG taper leading to a higher scattering loss. Therefore, the coupling efficiency of the PC taper is lower than that of the WG taper which is shown in Fig. 5(c). To include the affection of the enlarged air holes in the PC taper, an extra calculation has been performed. The result is more similar to the experimental results as shown in Fig. 5(b).

Fig. 5(c) shows the experimental results of the WG taper. The result is match to the simulation. The highest coupling efficiency is $74 \%$ for the taper length of $l_{c}=50 \mu \mathrm{m}(N A=0.32)$.

From the results above, for the shorter coupling length of $l_{c}=5 \sim 6 \mu \mathrm{m}(N A=2.24 \sim 2.03)$, the experimental coupling efficiencies of the graded PC coupler, the PC taper and the WG taper are around $34 \%\left(l_{c}=6 \mu \mathrm{m}\right), 16 \%\left(l_{c}=5 \mu \mathrm{m}\right)$ and $17 \%\left(l_{c}=5 \mu \mathrm{m}\right)(43 \%, 16 \%$ and $18 \%$ in theoretical result), respectively. Therefore, we can conclude that the graded PC coupler can offer a higher coupling efficiency in a short coupling length. For the parabolic mirror coupler, the coupling efficiencies of theoretical and experimental results are around 78\% and $48 \%$, respectively. From the calculation result by FDTD, we can find that the coupling efficiency of the parabolic mirror coupler is very sensitive to the spatial grid size (coupling efficiency is $78 \%$ when the grid size is $0.02 \mu \mathrm{m}$, but less than $50 \%$ when the grid size is $0.05 \mu \mathrm{m}$ ). Therefore, the difference between the theoretical and experimental results may caused by the roughness on the mirror surface which formed in fabrication process. Table I shows the highest coupling efficiencies and the corresponding coupling length for each coupler.
TABLE I

COMPARISON OF COUPLING EFFICIENCIES (THEORETICAL/EXPERIMENTAL, \%/\%)

\begin{tabular}{|c|c|c|c|c|}
\hline $\begin{array}{c}\text { Coupling } \\
\text { length } I_{C} \\
(\mu \mathrm{m})\end{array}$ & $\begin{array}{c}\text { Graded PC } \\
\text { coupler }\end{array}$ & PC taper & WG taper & $\begin{array}{c}\text { Parabolic } \\
\text { mirror } \\
\text { coupler }\end{array}$ \\
\hline 5 & $34 / 37$ & $16 / 16$ & $18 / 17$ & N.A. \\
\hline 6 & is $43 / 34$ & N.A. & N.A. & N.A. \\
\hline 20 & N.A. & to $40 / 38$ & $40 / 39$ & N.A. \\
\hline$\sim 26$ & N.A. & $25 / 25$ & 49 / 46 & ts $78 / 48$ \\
\hline 50 & N.A. & N.A. & is $71 / 74$ & N.A. \\
\hline
\end{tabular}

\section{CONCLUSION}

We studied the coupling between a dielectric WG with a width of $W_{c w}=10 \mu \mathrm{m}$ and a single-line-defect PCWG with a width of $738 \mathrm{~nm}$. The light source is TE polarized and the wavelength is $\lambda=1550 \mathrm{~nm}$. The original coupling efficiency is only $5.6 \%$ if the flat input WG ending directly connects to the PCWG. The coupling efficiencies of the graded PC coupler, PC taper, WG taper, and parabolic mirror coupler are calculated and measured. The coupling efficiencies of the graded PC coupler, PC taper and WG taper are $43 \%, 16 \%$, and $18 \%$ in theoretical result $(34 \%, 16 \%$, and $17 \%$ in experimental result) as the shorter coupling length is $l_{c}=5 \sim 6 \mu \mathrm{m}$ $(N A=2.24 \sim 2.03)$, respectively. The results show that the graded PC coupler can provide a higher coupling efficiency in a shorter coupling distance. In the case of parabolic mirror coupler, the higher coupling efficiency is obtained to be $78 \%$ in theoretical result (48\% in experimental result). However, the parabolic mirror coupler requires larger device surface for the $90^{\circ}$ light turning and lower surface roughness of the parabolic mirror.

The averaged back reflections of the graded PC coupler, PC taper, WG taper, and parabolic mirror coupler are $43 \%, 70 \%$, $15 \%$, and $6 \%$ in the theoretical results, respectively. The reflections are rather high, because the design of the couplers in this work is very simple. The innate faults of the couplers can be improved through additional improving designs [6], [8], [10]. The wider input waveguide may be another reason that increases the back reflection. For wider input waveguide, the back scattered EM waves produced on the interface between the PCWG and input WG can be easily coupled to the input waveguide and propagate in the reverse direction. This phenomenon is especially obvious for the graded PC coupler and PC taper because the interfaces producing the back scattered EM waves are close to the input WG or the back scattered EM waves cannot escape from the coupler in lateral direction. For all the couplers, the back reflection would be reduced by reducing the width of the input WG.

In [6], nonuniform PC tapers were studied. The design was not included in this work because the nonuniform PC tapers have an inborn fault. The nonuniform PC tapers modify the lattice structure. In the case of bigger scatterers (air holes in this work), the scatterers may be merged together and the photonic bandgap may be destroyed. In this paper, the ratio of the diameter of the air hole $D$ to the lattice constant $a$ is $0.86: 1$. In 
this condition, the deformation of lattice could easily cause the merging of the scatterers and reduce the ability of the confinement for EM waves.

In Fig. 5, some differences can be observed between the experimental and theoretical result. We speculated that the enlarged air holes in the PCWGs may be the cause of the difference between the experimental and theoretical results. This phenomenon is especially obvious in the case of PC taper because the PC taper is a PCWG with expanded terminal. The coupling efficiency of the PC taper with enlarged air holes was also calculated, and the result is shown in Fig. 5(b). The result is more similar to the experimental result. An unusual phenomenon can be observed when the coupling length is around $10 \sim 20 \mu \mathrm{m}$. The coupling efficiency of the PC taper with enlarged air holes is higher than the original PC taper.

Finally, we conclude that the graded PC is suitable for extremely short coupling length (high $N A$ ). For long coupling length (low NA), the WG taper may be more suitable to couple the EM waves into the PCWGs. For the same coupling length, the coupling efficiency of the pure PC tapers without any improving design is lower than that of the WG tapers in this study. The PC tapers might need additional optimization to increase the coupling efficiency. [6], [8] The parabolic mirror coupler requires low roughness of the mirror surface and larger device surface, but it can provide higher coupling efficiency and low back reflection.

\section{REFERENCES}

[1] S. Y. Lin et al., "Experimental demonstration of guiding and bending of electromagnetic waves in a photonic crystal," Science, vol. 282, no. 5387, pp. 274-6, Oct. 1998.

[2] P. E. Barclay et al., "Efficient input and output fiber coupling to a photonic crystal waveguide," Opt. Lett., vol. 29, no. 7, pp. 697-9, Apr. 2004.

[3] C. C. Chen et al., "Photonic crystal directional couplers formed by InAlGaAs nano-rods," Opt. Express, vol. 13, no. 1, pp. 38-43, Jan. 2005.

[4] W. Y. Chiu et al., "Directional coupler formed by photonic crystal InAlGaAs nanorods," J. Lightw. Technol., vol. 26, no. 5, pp. 488-491, Mar. 2008.

[5] W. Y. Chiu et al., "A photonic crystal ring resonator formed by SOI nano-rods," Opt. Express, vol. 15, no. 23, pp. 15500-15506, Nov. 2007.

[6] E. H. Khoo, A. Q. Liu, and J. H. Wu, "Nonuniform photonic crystal taper for high-efficiency mode coupling," Opt. Express, vol. 13, no. 20, pp. 7748-7759, Oct. 2005.

[7] T. D. Happ, M. Kamp, and A. Forchel, "Photonic crystal tapers for ultracompact mode conversion," Opt. Lett., vol. 26, no. 14, pp. 1102-1104, Jul. 2001.

[8] P. Sanchis et al., "Mode matching technique for highly efficient coupling between dielectric waveguides and planar photonic crystal circuits," Opt. Express, vol. 10, no. 24, pp. 1391-1397, Dec. 2002.

[9] E. Miyai et al., "Analysis of coupling between two-dimensional photonic crystal waveguide and external waveguide," Appl. Phys. Lett., vol. 81, no. 20, pp. 3729-3731, Nov. 2002.

[10] A. Mekis and J. D. Joannopoulos, "Tapered couplers for efficient interfacing between dielectric and photonic crystal waveguides," J. Lightw. Technol., vol. 19, no. 6, pp. 861-865, Jun. 2001.

[11] D. W. Prather et al., "High-efficiency coupling structure for a singleline-defect photonic-crystal waveguide," Opt. Lett., vol. 27, no. 18, pp. 1601-1603, Sep. 2002.

[12] H. T. Chien and C. C. Chen, "Focusing of electromagnetic waves by periodic arrays of air holes with gradually varying radii," Opt. Express, vol. 14 , no. 22 , pp. 10759-10764, Oct. 2006.
[13] K. Yee, "Numerical solution of initial boundary value problems involving maxwell's equations in isotropic media," IEEE Trans. Antennas Propag., vol. 14, no. 3, pp. 302-307, May 1966.

Hung-Ta Chien received the B.S. degree in electrical engineering from Yuan Ze University, Chung Li, Taiwan, in 2002 and the M.S. degree in optics science from National Central University (NCU), Chung Li, in 2004. Currently, he is working towards the Ph.D. degree at NCU.

His research interests include photonic crystal devices and micro-optical devices.

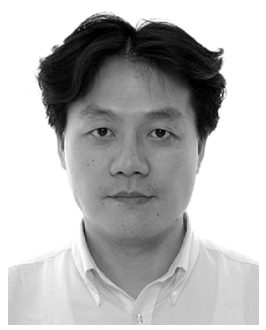

Chengkuo Lee (M'96) received the M.S. degree in materials science and engineering from National Tsing Hua University, Hsinchu, Taiwan, in 1991, the M.S. degree in industrial and system engineering from Rutgers University, New Brunswick, NJ, in 1993, and the Ph.D. degree in precision engineering from the University of Tokyo, Tokyo, Japan, in January 1996.

He was a Foreign Researcher in the Research Center for Advanced Science and Technology (RCAST), University of Tokyo, in 1996. He was also with the Mechanical Engineering Laboratory, AIST, MITI of Japan, as a Research Fellow in 1996. Thereafter, he was a Senior Research Staff of the Microsystems Laboratory, Industrial Technology Research Institute, Hsinchu, Taiwan. Since September 1997, he has been with the Metrodyne Microsystem Corporation, Hsinchu, Taiwan, and he established the MEMS Device Division and micromachining fabrication. He was the Manager of MEMS Device Division from 1997 to 2000. He was an Adjunct Assistant Professor in the Electro-Physics Department, National Chiao Tung University, in 1998, and an Adjunct Assistant Professor in the Institute of Precision Engineering, National Chung Hsing University, from 2001 to 2005. He cofounded Asia Pacific Microsystems, Inc., (APM) Hsinchu, Taiwan, in August 2001, and he became the Vice President (VP) of R\&D, then the VP of the Optical Communication Business Unit. Currently, he is an Assistant Professor at the Department of Electrical and Computer Engineering, National University of Singapore, and a Senior Member of Technical Staff at the Institute of Microelectronics (IME), Agency for Science, Technology, and Research (A*STAR), Singapore. He has contributed more than 160 international conference papers and international journal articles in the MEMS and nanotechnology fields.

Dr. Lee is a member of MRS and IEE Japan. He received the IUMRS Graduate Student Award in 1994.

Hua-Kung Chiu, photograph and biography not available at the time of publication.

Kuei-Chu Hsu, photograph and biography not available at the time of publication.

Chii-Chang Chen, photograph and biography not available at the time of publication.

Ja-an Annie Ho, photograph and biography not available at the time of publication.

Chien Chou, photograph and biography not available at the time of publication. 